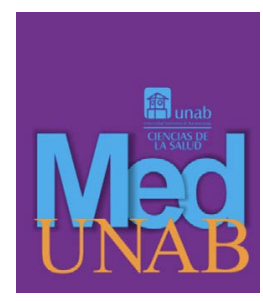

REVISTA DE LA FACULTAD

DE CIENCIAS DE LA SALUD

\title{
Síndrome de Burnout y la psiconeuroendocrinoinmunología: consecuencias en el personal de salud durante la pandemia por Coronavirus
}

Burnout syndrome and psycho neuro endocrino immunology: consequences in health workers during the Coronavirus pandemic

Síndrome de burnout e psiconeuroendocrinoimmunologia: consequências em profissionais de saúde durante a pandemia de Coronavírus

Jaime Alberto Urbiña-Villarraga, MD., Esp. ${ }^{1}$ iD, Sara Milena Velandia-Puerto, MD., Esp. ${ }^{2}$ (D), María Camila Gomez-Lora, MD., Esp. ${ }^{3}$ (D), David Steven Cañón-Ramirez, MD., Esp. ${ }^{4}$ (D), Tatiana Margarita María Vargas-Montoya, Esp. ${ }^{5}$ (D), David Humberto Harnache-Bustamante, MD,. Esp., MSc. ${ }^{6}$ (D)

1. Médico; Especialista en pediatría; Especialista en Epidemiología. Universidad del Sinú Elias Bechara Zainúm. Clínica Nueva de Cali, Clínica Colombia, Cali, Valle del Cauca, Colombia.

2. Médico; Especialista en Epidemiología. Fundación Universitaria Sanitas. Bogotá, Colombia.

3. Médico, Especialista en Epidemiología. Hospital Cardiovascular de Cundinamarca. Cundinamarca, Colombia.

4. Médico; Especialista en Epidemiología, Compensar EPS. Bogotá, Colombia.

5. Médico; Especialista en Epidemiología, Especialista en Gerencia en Salud. Centro dermatológico Angélica Guatibonza. Bogotá, Colombia.

6. Médico; Especialista en Ortopedia y Traumatología, Especialista en Epidemiología. Clínica San José, Barrancabermeja, Santander, Colombia.

Correspondencia. Jaime Alberto Urbiña Villarraga Clínica Nueva de Cali, Clínica Colombia, Cali.

Email: jurbina521@unab.edu.co

INFORMACIÓN DEL ARTÍCULO:

Artículo recibido: 01 de octubre 2020

Artículo aceptado: 04 de abril del 2021

DOI: https://doi.org/10.29375/01237047.3997

Cómo citar. Urbiña-Villarraga JA, Velandia-Puerto SM, Gomez-Lora MC, Cañón-Ramirez DS, Vargas-Montoya TMM, Harnache-Bustamante DH. Síndrome de Burnout y la psiconeuroendocrinoinmunología: consecuencias en el personal de salud durante la pandemia por Coronavirus. MedUNAB. 2021;24(1) 3997 doi: https://doi.org/10329375/01237047.3997 
Palabras clave: Agotamiento psicológico; Burnout, Salud mental; Infección por coronavirus; Pandemia.

Keywords: Psychological exhaustion; Burnout; Mental health; Coronavirus infection; pandemic.

Keywords: Esgotamento psicológico; Burnout; Saúde mental; Infecção por Coronavírus; Pandemia.

\section{Sr. Editor.}

Hasta la fecha se han documentado 7,439,220 de contagio por coronavirus (COVID-19) con una cifra de muertes de 961,400, de los cuales aproximadamente 7,000 hacen parte del personal de salud. En Colombia, las cifras por COVID-19 llegan a 758,398 con una mortalidad de 3,1\%, lo que equivale a 24,039 muertes. Esto ha significado una gran carga mental para el personal de salud, conduciendo a un incremento en el número de suicidios por parte de este personal.

Colombia no es ajena a la situación, tiene un reporte de 43,157 casos de los cuales 7,651 se dieron en médicos, 11,171 en auxiliares de enfermería, 4,611 en enfermeras profesionales. En cuanto a la mortalidad, hasta la fecha de revisión del 3 de febrero del 2021 había una cifra de 216 fallecidos de los cuales 71 eran médicos, 38 eran auxiliares de enfermería y trece enfermeras profesionales (1).

"Maslach y Jackson en 1981 definen el Síndrome de Burnout como un síndrome de agotamiento emocional, despersonalización y baja realización personal que puede ocurrir entre individuos que hacen trabajo en condiciones exigentes y estresantes que superan la capacidad de sus estrategias para afrontar sus expectativas"(2,3), esto ocasiona problemas de sueño, ansiedad depresión lo que conlleva a errores médicos y mala atención médica.

El Síndrome de Burnout es común en el personal que trabaja en una Unidad de Cuidados Intensivos, principalmente por la complejidad de los pacientes y la alta tasa de mortalidad en esta área. Pero, desde inicio del año 2020, cuando la Organización Mundial de la Salud (OMS) dio la alerta de pandemia del Coronavirus SARS-CoV-2, conocido como COVID-19, la vida misma ha cambiado de manera drástica.

El personal de salud, sin importar el área en la que se encuentra, se ha visto sometido a una carga laboral abrumadora debido al miedo que despierta la posibilidad de contagiarse y afectar a las personas de su alrededor, a su familia o a sus compañeros. Esto ha ocasionado separación de familias e incluso ha llevado a suicidios del personal de salud, como fue el caso de la enfermera italiana Daniela Trezzi, que fue publicado en casi todos los diarios del mundo. Como ella otros casos se presentaron.

En estudios previos realizados en Latinoamérica, en México y en Argentina, reportan cifras de personal médico con agotamiento de $4,9 \%$ hasta $16,8 \%$ entre médicos y enfermeras respectivamente. en el 2003 en Cuba, reportaron una prevalencia del $35 \%$ y el $23,7 \%$ para el personal médico y el personal de enfermería. En Barranquilla, Colombia en el 2006. muestran que un $1,9 \%$ del personal de enfermería presenta síndrome de desgaste, mientras que Cogollo et al. reportan un 26,6\% de desgaste en el personal de enfermería (4-7).

Monterrosa et al. (5), en el 2020, realiza un estudio transversal durante la pandemia de COVID-19, con una encuesta para la evaluación de síntomas de estrés y ansiedad en el personal médico. Usa la percepción sobre el COVID19 y tres escalas que fueron traducidas al español: escala del trastorno de ansiedad generalizada (GAD-7), fear COVID-19 scale (FCV-19S) y un cuestionario de problemas psicosomáticos. Los resultados muestran que el $40 \%$ de los encuestados refiere discriminación por la profesión ejercida y el $26 \%$ se siente alterado durante gran parte del día, con niveles de estrés leve en un 33,5\% de los encuestados, moderado en un $26 \%$ y severo en un $13,4 \%$. (5)

Más del $50 \%$ de los entrevistados están presentando un síntoma de ansiedad o estrés, lo que nos permite conocer las condiciones psíquicas de nuestro personal durante esta pandemia. Este tipo de investigaciones no solo deben ser realizadas al personal médico sino a todos quienes participan en las labores de cuidado en las instituciones de salud. La incidencia del Síndrome de Burnout varía dependiendo del estudio realizado y las escalas usadas. La importancia de continuar con este tipo de trabajos y mejorar las actividades radica en la posibilidad de distribuir mejor las cargas emocionales de los trabajadores de la salud. (5)

No solo basta con conocer el Síndrome de Burnout, es necesario ahondar en la alteración de la psiquis que puede conllevar a la pérdida de la homeostasis del cuerpo, predisponiendo a un mayor proceso inflamatorio y a la disminución de la efectividad del sistema inmunológico. Todo esto puede explicarse a la luz de la psiconeuroendocrinoinmunología, la ciencia que integran los cuatro sistemas involucrados en la estabilidad general del medio interno. Su estudio permite conocer de forma más integral el proceso saludenfermedad, ya que esta última es ocasionada por la alteración del equilibrio entre la psiquis y los diferentes 
sistemas del cuerpo. Este desequilibrio ocasiona daños en el organismo por su predisposición a la aparición de diversas enfermedades infecciosas y reumatológicas, causas de secreción continua y prolongada de citoquinas, corticotropina y cortisol. Todo esto es causado por exposición crónica al estrés, lo que conlleva a cambios estructurales en la corteza prefrontal, el hipocampo y la amígdala; deterioro cognitivo y mayor sensibilidad a nuevos agentes estresores $(8,9)$.

En tiempo de pandemia el estrés se ha elevado de manera exponencial en el personal de atención en salud de las diferentes áreas, lo que ha provocado una pérdida del equilibrio entre los cuatro sistemas anteriormente mencionados. Pero estos eventos no se han presentado exclusivamente durante esta pandemia, la alta carga de estrés en la población general desde años anteriores ha permitido que se considere al estrés como la pandemia del siglo XXI, relacionándose con la obesidad, la hipertensión arterial y la arteriosclerosis, entre otras patologías. Esto puede llevar a preguntarnos: ¿qué papel juega el Síndrome de Burnout en los desenlaces fatales del personal de salud y la población general sin aparentes afectaciones previas. (10).

Se deben realizar las escalas de la mejor forma posible para evaluar de forma constante el compromiso del personal, ya que se puede ver afectado dependiendo del donde ejerce su profesión. En estos momentos nadie está exento de contagio por parte del Coronavirus SARS$\mathrm{CoV}-2$, por lo que Montgomery et al. (3) recomiendan la implementación según el servicio afectado y que no se vea únicamente al sujeto sino a todo su entorno.

Se necesita mejorar las condiciones asistenciales al personal de salud, no poner más carga en sus hombros. La respuesta ha sido responsabilizar al personal para mejorar el cuidado y dejar que afronten solos los factores estresantes, lo que conlleva al desgaste y a sentimientos de ineficiencia ocasionados por el cansancio físico y mental del personal, más en estos momentos donde los pacientes fallecen por más que reciben todo el esfuerzo del personal.

En la literatura se citan seis aspectos críticos que llevan al agotamiento del personal de salud: altas demandas de trabajo junto con una falta de control, desconexión entre los valores individuales y los de la organización o sistema, recompensas insuficientes, sobrecarga de laboral, injusticias $\mathrm{y}$, por último, aislamiento de la comunidad (11).

Entre las intervenciones propuestas para disminuir el Síndrome Burnout del personal de salud se ha sugerido que el fortalecimiento de las relaciones entre los individuos y la institución es la clave para prevenirlo. En Colombia, este punto se ha consolidado como un problema como determinante social, las pobres infraestructuras, los malos pagos, la mala disposición de las instituciones hacia el personal de salud y la pobre intervención del gobierno ha llevado a una ruptura entre las instituciones y el personal.

La llegada de la vacunación a la población ha causado diversas opiniones. Ante los grupos antivacunas, el gremio de médicos y los gobiernos mundiales ponen su mayor esfuerzo para lograr una vacunación masiva teniendo como expectativa una solución para los estragos que causó la COVID-19, anhelando que esta llegue a su final. En uno de sus reportes el centro para el control y la prevención de enfermedades (con sus siglas en inglés CDC) muestra el esfuerzo que está realizando Israel para fortalecer su programa de vacunación masiva en personas de la tercera edad, muestra una eficacia para prevención de casos severos, un descenso de aproximadamente el $67 \%$ de estos en la población de más de 60 años (12).

Recomendamos la realización de intervenciones con formularios adecuados para la pronta identificación de factores de ansiedad y estrés en el personal de salud, estas intervenciones se deben realizar con periodicidad para optimizar la detección temprana. Es además de vital importancia optimizar las condiciones laborales del personal de salud en Colombia, mejorar las brechas de atención y trabajar sobre los efectos deletéreos que este agotamiento está causando en el gremio. Más aún cuando mientras se escribe este texto llegan noticias de amigos, colegas y docentes que están contagiados o que perdieron la dura batalla contra la enfermedad. Una enfermedad que tiene un componente social muy importante y que afecta directamente al gremio. Con la llega de la vacunación a nuestro país la esperanza se mantiene alta y el cuerpo médico, a través de campañas y redes sociales con los numerales \#yosimevacunocontraelcovid y \#porlosquenollegaronaestemomento, invitan a todo el pueblo a la vacunación, Para iniciar el control de este microorganismo que ha causado grandes pérdidas en los habitantes del mundo.

\section{Referencias}

1. Instituto Nacional de Salud Colombia. Coronavirus covid-19 en personal de salud en Colombia, Boletín. 2021; (66). Recuperado a partir de: https://www.ins.gov. co/Noticias/Paginas/coronavirus-personal-salud.aspx

2. Suñer-Soler R., Grau-Martín A., Flichtentrei D., Prats M., Braga F., Font-Mayolas S. et al. The 
consequences of burnout syndrome among healthcare professionals in Spain and Spanish speaking Latin American countries. Burnout Research. 2014; 1(2): 82-89. https://doi.org/10.1016/j.burn.2014.07.004

3. Montgomery A., Panagopoulou E., Esmail A., Richards T., Maslach C. Burnout in healthcare: the case for organisational change. BMJ. 2019; 366: 14774. https://doi.org/10.1136/bmj.14774

4. Hernández-Vargas CI, Juárez-García A, HernándezMendoza E, Ramírez-Páez JA. Burnout y síntomas somáticos cardiovasculares en enfermeras de una institución de salud en el Distrito Federal. Rev Enferm IMSS 2005; 13: 125-31.

5. Monterrosa-Castro A, Dávila-Ruiz R, Mejía-Mantilla A, Contreras-Saldarriaga J, Mercado-Lara M, FloresMonterrosa C. Estrés laboral, ansiedad y miedo al COVID-19 en médicos generales colombianos. MedUNAB. 2020; 23(2): 195-213. https://doi. org $/ 10.29375 / 01237047.3890$

6. Tuesca-Molina R, Iguarán M, Suárez M, Vargas G, Vergara D. Síndrome de desgaste profesional en enfermeras/os del área Metropolitana de Barranquilla. Salud Uninorte 2006; 22: 84-9

7. Cogollo-Milanés, Z., Batista, E., Cantillo, C., Jaramillo, A., Rodelo, D., \& Meriño, G. (2010). Desgaste profesional y factores asociados en personal de enfermería de servicios de urgencias de Cartagena,
Colombia. Aquichan, 10(1). Recuperado a partir de https://aquichan.unisabana.edu.co/index.php/ aquichan/article/view/1605

8. Padrón González AA, Martínez Infante A. Estrés, psiconeuroendocrinoinmunología y enfermedades reumatológicas. Actualización del tema. Rev Cubana de Reumatol. 2018; 20(3). https://dx.doi.org/10.5281/ zenodo.1469002

9. Oken BS, Chamine I, Wakeland W. A systems approach to stress, stressors and resilience in humans. Behav Brain Res. 2015; 282: 144-154. https://doi. org/10.1016/j.bbr.2014.12.047

10. Arteaga-Roman A, Junes-Gonzales W, NavarreteSaravia A. Prevalencia del Síndrome de Burnout en personal de Salud. Rev méd panacea. 2014; 4 (2): 4044.

11. Aldrete Rodríguez MG, Navarro Meza C, González Baltazar R, Contreras Estrada MI, Pérez Aldrete J. Psychosocial factors and burnout syndrome in nurses of a unit of tertiary health care. Cienc Trab. 2015; 17(52): 32-36. https://doi.org/10.4067/S071824492015000100007

12. Rinott E, Youngster I, Lewis E. Reduction in COVID-19 Patients Requiring Mechanical Ventilation Following Implementation of a National COVID-19 Vaccination Program - Israel. MMWR. 2021; 70. https://doi.org/10.15585/mmwr.mm7009e3 